\title{
Optimal surgical procedures for traumatic blunt duodenal injury: A single-center experience
}

\author{
Takahiro Murakami*, Morihiro Katsura, Masafumi Ie, Takashi Kato \\ Okinawa Chubu Hospital, Department of Surgery, 281 Aza Miyazato Uruma-shi, Okinawa 904-2243, Japan
}

\begin{abstract}
Background: Blunt traumatic injury of the duodenum is relatively rare, but it is known that early diagnosis and treatment are essential to increase survivability. A variety of ideas have been put forth regarding optimal management of blunt duodenal injury, but more information from outcomes of completed procedures by experienced surgeons needs to be reported to facilitate the improvement of guidelines and patient care. In this study, we review results from our hospital and share what has been learned from our experience in the management of blunt traumatic injury of the duodenum.
\end{abstract}

Methods: A retrospective chart review of 19 patients with blunt duodenal injuries who were treated in a single institution from 1990 to 2018 was conducted. Cases of intramural hematoma of the duodenum were excluded.

Results: Fifteen (58\%) individuals had been involved in motor vehicle accidents. GradeII duodenal injury was the most observed type of injury (74\%). The second portion of the duodenum was the most commonly injured, and there was a high incidence of associated intra-abdominal injuries. Simple closure was performed in 14 cases. Duodenal resection and anastomosis were performed in 4 cases, without pyloric exclusion or duodenal diverticulization. Duodenal fistula was observed in only one case $(6.3 \%)$, and there were no cases of duodenal injury-related death (0\%).

Conclusions: Simple repair without complicated additional procedures (pyloric exclusion and duodenal diverticulization) may be adequate and safe for most blunt duodenal injuries.

\begin{abstract}
Abbreviations: AAST-OIS: American Association for the Surgery of Trauma organ injury scale; ISS: Injury Severity Score; CT: Computed Tomography; PD: Pancreaticoduodenectomy
\end{abstract}

\section{Background}

Traumatic duodenal injury is a relatively rare condition comprising less than $5 \%$ of all traumatic abdominal injuries [1]. It is usually caused by blunt abdominal trauma rather than penetrating injury, especially in Japan. Blunt duodenal injury is also uncommon, accounting for less than $1 \%$. It is mainly caused from direct compression between two opposing surfaces - the abdominal wall and the spine. This leads to increased risk of injury and tearing in fixed areas of the bowel, such as the duodenum [2]. Morbidity related to duodenal injuries varies with reported rates ranging from $7 \%$ to $55 \%$, while mortality rate has been reported to range from $5 \%$ to $23 \%$ [3-5]. Currently, injury to the duodenum presents a significant challenge for trauma surgeons. Using the American Association for the Surgery of Trauma organ injury scale (AAST-OIS), duodenal injuries are graded from I-V in ascending order of severity with treatment and therapeutic plan guided by the injury severity [6]. However, in most clinical settings, the degree of injury, abdominal contamination, and the patient's condition, especially in blunt injury, are extremely diverse. There is no unified view regarding the optimal surgical treatment for all scenarios. At our institution, appropriate debridement, suture closure, and resection/anastomosis of the injured section are considered the most important aspects of the surgical operation, and complicated additional procedures (pyloric exclusion and duodenal diverticulization) are considered unnecessary as a general rule [7]. This study investigated the validity of this operative mindset.

\section{Methods}

We conducted a retrospective cohort study between 1990 and 2018; in our hospital using data extracted from patient charts. We reviewed all all patients with duodenal injury by using trauma registry system of our institution. Those with grade I injury, which is managed non-surgically, and penetrating duodenal injury were excluded to limit the search to patients who had full-thickness blunt duodenal injury. This established the subset for the study. Standard demographic and descriptive data were collected for all patients, including age, sex, mechanism of injury, vital signs on admission, physical examination on admission, diagnostic modalities and results, Injury Severity Score (ISS), timing and findings of operation, type of operation (simple closure, resection and anastomosis, pancreaticoduodenectomy (PD)), and clinical outcomes (including mortality, cause of death, complications, length of stay, and disposition). As this study was a chart review and was of no risk to subjects, the need for informed consent from each patient was waived per decision of the institutional review board of Okinawa Chubu Hospital. Further, as no individual identifying data was contained in this study, individual consent was not applicable.

${ }^{*}$ Correspondence to: Takahiro Murakami, Okinawa Chubu Hospital, Department of Surgery, 281 Aza Miyazato Uruma-shi, Okinawa 904-2243, Japan, Tel. 81-098-973-4111; Fax: 81-098-9735165; E-mail: murakami_takahiro@hosp. pref.okinawa.jp

Key words: blunt duodenal injury, simple repair, duodenal fistula, mortality

Received: October 28, 2020; Accepted: November 24, 2020; Published: November 27, 2020 


\section{Results}

A total of 38 patients with traumatic duodenal injuries were admitted to our hospital during the study period. Of these, 11 patients with grade I injuries who had been treated with conservative treatment were identified and excluded from analysis. Eight patients with penetrating duodenal trauma were also excluded. A final total of 19 surgical patients were included in the analysis.

Patient background data are summarized in Table 1 . The average age was 29 years (range, 1-72 years) with a male/female ratio of 15:4. The average ISS was 21 (range, 11-48). Traffic-related injuries were the most common cause of injury and were noted in 15 patients, 12 of whom were either vehicle drivers or passengers.

Two patients were injured in industrial accidents (fall and being caught in machinery) and 2 additional patients were injured during domestic violence incidents.

Treatment guidelines and surgical indications for blunt duodenal injury at our hospital are shown in Figure 1. These are based on the current AAST management algorithm for duodenal injury [4]. Of the 19 patients reviewed, 6 (32\%) did not respond to initial resuscitation for trauma and had to undergo emergency laparotomy for hemostasis due to intra-abdominal bleeding. In these patients, the duodenal injury was diagnosed during surgery. The remaining 13 patients responded to initial resuscitation for trauma and were subjected to a thorough examination after stabilization of vital signs. Peritonitis was later diagnosed in all these cases, and laparotomy was performed. Of these, surgeries were performed on 3 patients without conducting abdominal computed tomography (CT) scans. Scans were conducted on the remaining 10 patients before surgery. However, intra- and retroperitoneal free air could be observed in only 5 of these cases (50\%), and a preoperative diagnosis of duodenal injury could only be made in 5 patients $(38 \%)$. The average time from admission to surgery was $5.7 \mathrm{~h}$ (range, $55 \mathrm{~min}$ $18 \mathrm{~h}$ ), with all patients being diagnosed and treated within $24 \mathrm{~h}$.

Similar to previous reports, D2 (descending) and D3 (horizontal) were the most common sites of duodenal injury in our review [8]. Four patients presented with D1 (duodenal bulb), 8 presented with D2, five presented with D3, and 2 presented with D4 (from the superior mesenteric artery/vein to the ligament of Treitz) injuries. Furthermore, 2 patients had multiple sites of injury. The most common severity of injury was grade II, found in 14 patients (74\%), followed by grade III, found in 4 patients. Grade V severity was observed in 1 patient.

Additional injuries involving other organs were observed in $100 \%$ of the patients (Table 2). Among these, liver injury was the most common comorbidity, present in 11 patients, followed by colon and pancreatic injuries observed in 6 and 5 patients, respectively. Comorbid pancreas injuries included 4 grade II injuries and 1 grade IV injury. Several patients also sustained severe comorbid vascular injuries - one

Table 1. Patient demographics

\begin{tabular}{|l|c|}
\hline Age in mean years (range) & $29(1-72)$ \\
\hline Sex (male) & $15(79 \%)$ \\
\hline ISS (range) & $21(11-48)$ \\
\hline Mechanism & - \\
\hline Motor vehicle crash & 11 \\
\hline Cyclist struck & 2 \\
\hline Pedestrian struck & 1 \\
\hline Motor cyclist & 1 \\
\hline Industrial accident & 2 \\
\hline Domestic violence & 2 \\
\hline
\end{tabular}

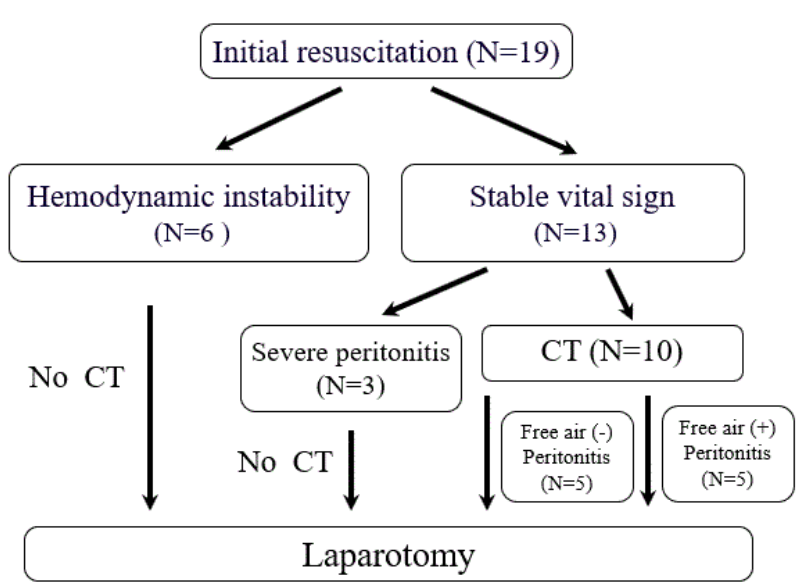

Figure 1. Management Strategy

Table 2. Associated injuries

\begin{tabular}{|l|c|}
\hline Abdominal injuries & $\mathbf{2 2}(\mathbf{8 1} \%)$ \\
\hline Liver & $\mathbf{1 2}$ \\
\hline Pancreas & 6 \\
\hline Portal triad & 2 \\
\hline Stomach & 1 \\
\hline Small intestine & 5 \\
\hline Large intestine & 9 \\
\hline Kidney & 4 \\
\hline Mesentery & 3 \\
\hline IVC & 3 \\
\hline SMA/SMV & 1 \\
\hline Common iliac artery & 1 \\
\hline Extra-abdominal injury & $17(63 \%)$ \\
\hline
\end{tabular}

IVC: inferior vena cava

SMA: superior mesenteric artery

SMV: superior mesenteric vein

involving the inferior vena cava, 2 involving the portal triad, 1 involving the superior mesenteric arteriovenous base, and 1 with comorbid iliac artery injury.

The surgical procedures performed and their outcomes are shown in Table 3. Debridement with simple suture closure was performed at the site of injury for 14 patients. Of these, an omental or serosal patch was added in 5 cases. Resection and anastomosis was performed in 4 patients. The methods of anastomosis were as follows: duodenal end-to-end anastomosis in 2 patients; duodenal jejunal side-to-side anastomosis in 1 patient; and duodenal jejunal Roux-en-Y anastomosis in 1 patient. A single-stage PD was performed on one patient who experienced grade $\mathrm{V}$ multiple duodenal injuries together with comorbid grade IV pancreatic head injuries. Duodenal fistula, likely due to suture insufficiency, was observed in 1 patient, with a recorded suture insufficiency rate of $6.3 \%$ (1/16 patients), after excluding deaths and the patients who underwent PD. This patient had grade III duodenal injury at D3 with SMA/SMV injury and grade II pancreas injury (ISS27). Duodenal resection and end-to-end anastomosis had been performed, and the time from injury to operation was $140 \mathrm{~min}$. This patient had duodenal fistula and SMA pseudoaneurysm formation treated by drainage and interventional stent placement. The patient recovered well and was finally discharged on hospital day 279 . There were two deaths, resulting in a mortality rate of $10.5 \%$. Both patients had multiple severe injuries with an ISS of 34 and 48, respectively both died within $24 \mathrm{~h}$ of surgery due to hemorrhagic multiple organ failure. No deaths were associated with duodenal injury or suture insufficiency. 
Table 3. Operative management and outcomes

\begin{tabular}{|c|c|c|c|c|c|c|}
\hline Operation & Number & Wound infection & Abscess & Fistula & Length of stay (days) & Mortality \\
\hline Primary closure with patch & 8 & 1 & 2 & 1 & 30 & $\mathbf{0}$ \\
\hline Primary closure without patch & 12 & 1 & 1 & $\mathbf{0}$ & 26 & 2 \\
\hline Roux en-Y anastomosis & 1 & $\mathbf{0}$ & $\mathbf{0}$ & $\mathbf{0}$ & 14 & $\mathbf{0}$ \\
\hline Resection + end to end anastomosis & 3 & 1 & 1 & 1 & 82 & $\mathbf{0}$ \\
\hline Resection + side to side anastomosis & 2 & $\mathbf{0}$ & 1 & $\mathbf{0}$ & 28 & $\mathbf{0}$ \\
\hline Pancreaticoduodenectomy (PD) & 1 & 1 & 1 & 1 & 102 & $\mathbf{0}$ \\
\hline
\end{tabular}

\section{Discussion}

In this study, we obtained good results in the surgical management of blunt duodenal injuries using only appropriate debridement, suture closure, and resection/anastomosis, without complicated additional procedures.

The treatment of traumatic duodenal injuries often not only involves a delay in the diagnosis owing to the retroperitoneal location of the duodenum, but may also involve complicated injuries to the surrounding organs, making subsequent surgical decisions difficult [9]. Therefore, early diagnosis and surgery within the initial $24 \mathrm{~h}$ are essential to increase survival [10].

Abdominal trauma that does not respond to initial resuscitative actions and is accompanied by massive intraperitoneal bleeding is an indication for immediate laparotomy. Injuries to each involved organ diagnosed during emergency surgery should be performed after suitable hemostatic control. Emergency laparotomy was performed without a CT scan in $32 \%$ of patients in this study, with the duodenal injuries diagnosed intraoperatively. During laparotomy, confirming the presence of intraperitoneal bleeding or duodenal injury should not be the sole objective. The presence of intestinal fluid, bile, air, or blood in the retroperitoneal space should also be considered [9].

We also recorded medical history and performed physical examination as a matter of routine in patients who responded well and had stable vital signs after the initial resuscitation. In cases of retroperitoneal injury, patients often exhibit only mild abdominal distension and tenderness, no rebound pain, and abdominal guarding in the early stages of injury, with symptoms and findings worsening gradually [2]. Therefore, it is important to perform physical examinations regularly and to review findings over time. Abdominal contrast-enhanced CT is the fastest and most comprehensive diagnostic imaging modality for these cases. Typical findings include intra- and retroperitoneal free air and images of hematoma and edema in the duodenal vicinity and retroperitoneum [9]. In a recent study, CT had a sensitivity and specificity of $86 \%$ and $88 \%$, respectively, in diagnosing blunt hollow viscus injury [11]. However, missed blunt duodenal injury rates of up to $27 \%$ have been described [12]. Intraperitoneal or retroperitoneal extraluminal air is a relatively specific sign of bowel perforation seen in $20-55 \%$ of patients. However, this may not be visible immediately after traumatic perforation [13]. In our study, the detection rate of intra-/retroperitoneal free gas using CT images was 50\%, with only 5 patients diagnosed with duodenal injuries prior to surgery. Thus, it can be concluded that though abdominal CT can be attempted in patients with stable vital signs, it is not perfect. Careful interpretation and clinical correlation are mandatory to avoid delayed diagnosis, treatment, and increased rates of case morbidity and mortality [9].

Postoperative duodenal fistulas, likely due to suture insufficiencies, have been a major concern in the management of traumatic duodenal injuries. This is due to anatomical and physiological characteristics which may lead to a need for complicated additional procedures, such as duodenal diverticulization [14] and pyloric exclusion [15], in order to overcome this problem. Though numerous facilities have stressed the importance of these procedures in the management of traumatic duodenal injury [16], recent reports from western countries have questioned their effectiveness [17-19]. In addition, there is currently a trend towards less invasive procedures for duodenal injury, as noted by a retrospective analysis of the National Trauma Data Bank of the United States from 2002 to 2014 . There it was noted that in-hospital mortality has improved as a result [20]. At our institution, appropriate debridement, suture closure, and resection/anastomosis of the injured section are considered to be the most important aspects in surgical management for blunt duodenal injuries, with complicated additional surgeries considered unnecessary as a general rule [7]

Our group prefers simple suture closures for grade II injuries that do not exceed half the circumference of the duodenum, with omental or serosal patches added as needed, depending on the tissue tension and extent of blood flow availability. There are no clear-cut indications for patch addition, so these are used at the discretion of the surgeon. Decompression of the duodenum with a nasogastric (NG) tube is not routinely performed in our hospital. Lateral-end anastomosis at the jejunal Roux-en-Y leg is performed for injuries that exceed half of the circumference of the duodenum and where tissue blood flow is relatively maintained. Partial duodenal resection and anastomosis are performed in grade III injuries that exceed half of the duodenal circumference and exhibit poor blood flow. The method of anastomosis (end-to-end or side-to-side) is selected based on the injury site, tissue tension, and blood flow status. The suture insufficiency rate at our facility was $6.3 \%$, with surgeries being performed as per the stated guidelines, and the mortality rate due to duodenal injuries was $0 \%$. Roman et al. reported a suture insufficiency and mortality rate of $25 \%$ and $5 \%$, respectively, for surgeries conducted within $24 \mathrm{~h}$, whereas the mortality rate for surgeries after $24 \mathrm{~h}$ was $65 \%$ (4). Ballard et al. also reported that the mortality rate related to duodenal injuries was $7 \%$ (5). The results obtained at our facility compared favorably with those observed in these studies.

A single-stage PD was performed in patients with complications from GradeVduodenal injuries who were in stable condition during the perioperative period. However, pancreatic fistula and intraperitoneal abscesses developed in one patient after surgery, and that individual had to undergo long-term hospitalization for 102 days postoperatively. Second-stage surgeries that combine damage control surgery have been recommended by current guidelines as a treatment strategy for GradeVduodenal injuries with unstable vital sign and obvious intraabdominal contamination [21]. Although the concept of damage control surgery is universally accepted, if initial vital signs are stable and obvious contamination is not recognized, it is not easy to determine the optimal surgical procedure for grade $\mathrm{V}$ duodenal injury. This is because there is no unified view on this subject. For these types of complex cases, the general condition of the patient, the site and extent of injury, the type of accompanying injury, the extent and repair status, and the facility, as well as the surgeon's experience and skill level, need to be comprehensively determined. Only then can a surgical procedure 
that is most appropriate for the moment and location be decided. As it stands, there is no standard procedure for such cases.

In this study, with the exception of the patient who underwent $\mathrm{PD}$, the single patient who developed suture insufficiency did not have particularly high ISS values, and exhibited stable vital signs during the perioperative period. On further scrutiny, this patient had comorbid injury of the superior mesenteric arteriovenous base and pseudoaneurysm formation. This might lead to insufficient blood flow to the duodenum and cause insufficient anastomosis healing. Furthermore, the two deaths observed involved patients with multiple severe traumatic injuries and ISS values of 43 and 48 , respectively. Both patients had portal triad injury complications, with intraoperative hemorrhaging that was extremely difficult to control. Both died within $24 \mathrm{~h}$ of the surgery due to hemorrhagic multiple organ failure.

As described above, we witnessed favorable results at our facility with respect to our surgical protocol for traumatic duodenal injuries without the need for additional procedures. However, several limitations of the present study should be mentioned. The role of the following factors in obtaining favorable results is important: 1 ) in our cohort, there were a majority of simple injuries that were grade II in severity; 2) all patients underwent initial staging, diagnosis, and treatment within $24 \mathrm{~h} ; 3$ ) only 1 patient had grade $\mathrm{V}$ duodenal injury with severe pancreatic head disruption.

In the future, application, along with investigation, of additional complicated procedures should be considered in a flexible manner for high risk patients in poor general condition, or for those with significant injuries, intraperitoneal contamination, iatrogenic duodenal injuries, or in cases of duodenal perforation due to malignant tumor infiltration. However, additional procedures may incur complications, such as the formation of gastrointestinal ulcers and bleeding. Therefore, surgeons should avoid performing them unless deemed necessary. We plan to conduct further studies once we have accumulated additional cases with a greater number of established indications.

\section{Conclusions}

In this study, we observed favorable results at our facility after conducting surgeries for blunt duodenal injuries, performing only appropriate debridement, suture closure, and resection/anastomosis of the injured section, without the need for additional complicated procedures. Despite advances in diagnostic and surgical techniques, duodenal injury remains a challenging condition with high morbidity and mortality. Further prospective studies with larger cohorts that incorporate advances in surgical management are warranted.

\section{Acknowledgements}

Not applicable.

\section{Funding}

Not applicable.

\section{Ethics declarations}

\section{Ethical considerations}

Data collection for this study was approved by the institutional review board of our hospital, which is a review board that follows the principles set by the Declaration of Helsinki.

\section{Consent for publication}

As this study was a chart review and was of no risk to subjects, the need for informed consent from each patient was waived per decision of the institutional review board of Okinawa Chubu Hospital. Further, as no individual identifying data was contained in this study, individual consent was not applicable.

\section{Competing interests}

The authors declare that they have no competing interest.

\section{References}

1. Asensio JA, Feliciano DV, Britt LD (1993) Management of duodenal injuries. Curr Probl Surg 30: 1023-1093. [Crossref]

2. Williams RD, Sargent FT (1963) The mechanism of intestinal injury in trauma. $J$ Trauma 31: 735-748.

3. Blocksom JM, Tyburski JG, Sohn RL, Williams M, Harvey E, et al. (2004) Prognostic determinants in duodenal injuries. Am Surg 70: 248-55.

4. Roman E, Silva YJ, Lucas C (1971) Management of blunt duodenal injury. Surg Gynecol Obstet 132: 7-14.

5. Ballard RB, Badellino MM, Erynon CA, Spott MA, Staz CF, et al. (1997) Blunt duodenal rupture: A 6-year statewide experience. J Trauma 43: 229-32.

6. Moore EE, Cogbill TH, Malangoni MA, Jurkovich GJ, Champion HR, et al. (1990) Organ injury scaling II: Pancreas, duodenum, small bowel, colon, and rectum. J trauma 30: $1427-1429$.

7. Cogbill TH, Moore EE, Feliciano DV, Hoyt DB, Jurkovich GJ, et al. (1990) Conservative management of duodenal trauma: A multicenter perspective. J Trauma 30: 1469-75. [Crossref]

8. Resnicoff SA (1967) Retroperitoneal rupture of the duodenum due to blunt trauma Surg Gynecol Obstet 125: 77. [Crossref]

9. Walter L (2011) Duodenum and pancreas. In: Mattox KL, Feliciano DV, Moore EE, editors. Trauma. 7th ed. New York: McGraw-Hill; 603-619.

10. Lucus CE, ledgerwood AM (1975) Factors influencing outcome after blunt duodenal injury. J Trauma 15: 839-846. [Crossref]

11. Choi AY, Bodanapally UK, Shapiro B, Patlas MN, Katz DS (2018) Recent advances in abdominal trauma computed tomography. Semin Roentogenol 53: 178-186. [Crossref]

12. Allen GS, Moore FA, Cox CS, Mehall JR, Duke JH (1998) Delayed diagnosis of blunt duodenal injury: an avoidable complication. J Am Coll Surg 187: 393-399. [Crossref]

13. Brofman N, Atri M, Hanson JM, Grinblat L, Chughtai T, et al. (2006) Evaluation of bowel and mesenteric blunt trauma with multidetector CT. Radiographics 26: 1119-1131.

14. Berne CJ, DonovanAJ, White EJ, Yellin AE (1974) Duodenal “diverticulization" for duodenal and pancreatic injury. Am J Surg 127: 503-507. [Crossref]

15. Vaughan GD III, Frazier OH, Graham DY, Mattox KL, Petmecky FF, et al (1977) The use of pyloric exclusion in the management of severe duodenal injuries. Am J Surg 134: 785-790. [Crossref]

16. Synder WH, Weigelt JA, Watkins WL, Bietz DS (1980) The surgical management of duodenal trauma. Precepts based on a review of 247 cases. Arch Surg 115: 422-429. [Crossref]

17. Seamon MJ, Pieri PG, Fisher CA, Gaughan J, Santora TA, et al. (2007) A tenyear retrospective review: Does pyloric exclusion improve clinical outcome after penetrating duodenal and combined pancreaticoduodenal injuries? J Trauma 62: 829833. [Crossref]

18. Velmahos GC, Constantinou C, Kasotakis G (2008) Safety of repair for severe duodenal injuries. World J Surg 32: 7-12.

19. DuBose JJ, Inaba K, Teixeira PG, Shiflett A, Putty B, et al. (2008) Pyloric exclusion in the treatment of severe duodenal injuries: results from National Trauma Data Bank. Am Surg 74: 925-929. [Crossref]

20. Aiolfi A, Matsushima K, Chang G, Bardes J, Strumwasser A, et al. (2019) Surgical trend in the management of duodenal injury. J Gastroint Surgery 23: 264-269. [Crossref]

21. Coccolini F, Kobayashi L, Kluger Y, Moore EE, Ansaloni L, et al. (2019) Duodenopancreatic and extrahepatic biliary tree trauma: WSES-AAST guidelines. $W J$ Emerg Surgery 14: 56 .

Copyright: (C2020 Murakami T. This is an open-access article distributed under the terms of the Creative Commons Attribution License, which permits unrestricted use, distribution, and reproduction in any medium, provided the original author and source are credited. 